\title{
Cowbree: A novel dataset for fine-grained visual categorization
}

\author{
Umar Akbar Khan ${ }^{1}$, Saira Moin U. Din ${ }^{2}$, Saima Anwar Lashari ${ }^{3}$, \\ Murtaja Ali Saare ${ }^{4}$, Muhammad Ilyas ${ }^{5}$ \\ ${ }^{1,2}$ Department of Computer Science, the University of Lahore, Sargodha, Pakistan \\ ${ }^{3}$ College of Computing and Informatics, Saudi Electronic University, Dammam, Saudi Arabia \\ ${ }^{4}$ Universiti Utara Malaysia, Kedah, Malaysia \\ ${ }^{5}$ Department of CS \&IT, University of Sargodha, Pakistan
}

\begin{tabular}{|c|c|}
\hline Article Info & ABSTRACT \\
\hline Article history: & Fine-grained visual categorization (FGVC) dealt with objects belonging to \\
\hline Received Dec 14, 2019 & $\begin{array}{l}\text { one class with intra-class differences into subclasses. FGVC is challenging } \\
\text { due to the fact that it is very difficult to collect enough training samples. }\end{array}$ \\
\hline Revised Feb 22, 2020 & This study presents a novel image dataset named Cowbreefor FGVC. \\
\hline Accepted Apr 3, 2020 & $\begin{array}{l}\text { Cowbree dataset contains } 4000 \text { images belongs to eight different cow breeds. } \\
\text { Images are properly categorized under different breed names (labels) based }\end{array}$ \\
\hline Keywords: & $\begin{array}{l}\text { on different texture and color features with the help of experts. While } \\
\text { evidence shows that the existing dataset are of low quality, targeting few }\end{array}$ \\
\hline $\begin{array}{l}\text { Cowbree } \\
\text { Fined-grained visual } \\
\text { categorization } \\
\text { J48 }\end{array}$ & $\begin{array}{l}\text { breeds with less number of images. To validate the dataset, three state of } \\
\text { the art classifiers sequential minimal optimization (SMO), Multiclass } \\
\text { classifier and J48 were used. Their results in term of accuracy are } 68.81 \% \text {, } \\
55.81 \% \text { and } 57.45 \% \text { respectively. Where results shows that SMO out } \\
\text { performed with } 68.81 \% \text { accuracy, } 68.4 \% \text { precision and } 68.8 \% \text { recall. }\end{array}$ \\
\hline
\end{tabular}

Multiclass

SMO

WEKA image filter package

\section{Corresponding Author:}

Saira Moin U. Din,

Department of Computer Science,

The University of Lahore,

10 KM, Lahore Road, Sargodha, Pakistan.

Email: saira.moin@cs.uol.edu.pk
This is an open access article under the CC BY-SA license.

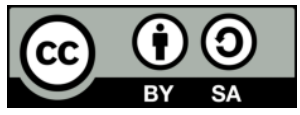

\section{INTRODUCTION}

Classification task performed without deep knowledge of the domain is always challenging. It is more difficult when there are specific intra-class differences between objects. In Computer vision Fine-grained visual categorization (FGVC) refers to categorize the objects belonging to one class but having the intra-class differences into subclasses. Fine-grained visual categorization (FGVC) is challenging because, it is often difficult to acquire enough number of training samples [1-2]. The existing datasets in this scenario have few training samples in each category. Few benchmark datasets like Stanford dogs [3] and Veg Fru [4] can be considered complete for this scenario, as these datasets have large number of training examples in each defined category. Collection and classification of data for specific category of object is a very challenging task. It requires deep domain knowledge and experience. For this particular reason datasets for FGVC is very limited.

Therefore this study aims to build a novel domain-specific dataset of cow breeds for image fined grained visual categorization. It is very difficult to differentiate between the different breeds of cow, due to very minute intra-class differences among them. Like it is very difficult to distinguish Red Sindhi from Sahiwal cow breed. Both have almost same texture and color features. Only the difference between them is their head shape. Figure 1 shows the example of Sahiwal and Red Sindhi cow breed. 
Here, it is mandatory to mention that biological categories-species and breeds-have been especially well-studied, with work tackling subcategory recognition of flowers, dogs, birds, etc. These biological domains, where taxonomy dictates a clear set of mutually exclusive subcategories, are wonderfully well-suited to the problem, and recognition systems in these domains are of practical use in ecology and agriculture. In this work mainly eight famous cow breeds are targeted, the dataset contains 4000 images belonging to eight different breeds of cow.
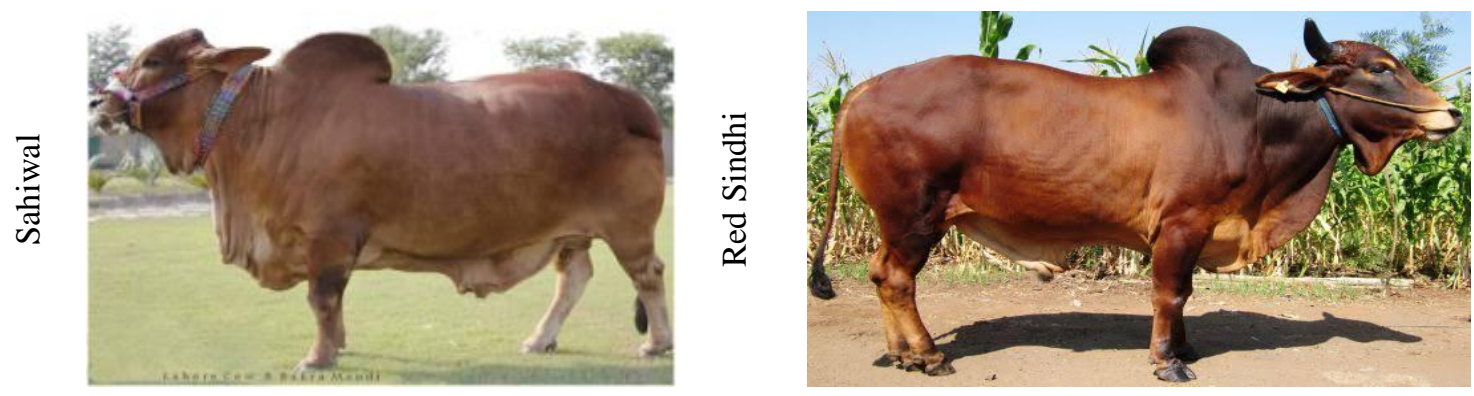

Figure 1. Example of sahiwal and red sindhi

a. Cow breeds

Livestock appears as natural machine for the production of dairy items and meat. It is quite fact that proteins from animals are much superior to proteins from vegetables. Livestock plays essential part in economic growth for the countries that mainly depend on agriculture. They provide social security in case of crop failure or in case of any other natural disaster. Livestock is an important sub-sector of the agriculture system and responsible for the economic growth of many Asian countries. According to a survey, in 2013 Asian countries produce $39 \%$ of world's cow milk and $97 \%$ of world's buffalo milk [5]. About half of the world's poor population lives in South Asia and they mainly depend on livestock for their social-economic growth [6-7]. Only in Pakistan round about 35 million people are engaged with the field of livestock, which show that livestock is an important and essential part of social system. Livestock involves different types of animals from a very large size camel to a small size goat but the basic purpose of all types of domestic animals is the production of meat and milk. Selection of breed is an important factor in this business. It is much difficult task and needs very deep knowledge. As many cow breeds are much similar in shape and color, like Red Sindhi and Sahiwal. Red Sindhi and Sahiwal are two different breeds with different milk production values but in case of color and shape these breeds are quite similar. This problem of breed recognizing can be solved by technology.

This study focuses on cow breeds. In many countries cow farming is a very large business, selection of breed is an important factor in this business. It is much difficult task and needs very deep knowledge. As many cow breeds are much similar in shape and color, like Red Sindhi and Sahiwal. Red Sindhi and Sahiwal are two different breeds with different milk production values but in case of color and shape these breeds are quite similar. This problem of breed recognizing can be solved by technology.

Now a day systems are available for automatic detection of animals and birds breed. Like Merlin Bird Photo ID an android app build by computer vision researchers for automatic detection of round about 400 species of birds in North America. Typically for detection of cow breeds no such type of system available in market. This study road towards that system; any breed detection system involves two parts one is building a dataset to train the model; other is the development of model that can be properly trained to recognize the different classes of objects. Like Zalán Ráduly et al., use benchmark dataset of Stanford dog breeds for the development of a dog breed identification model [8]. This study basically targets the first part i.e. building a cow breed dataset. One of the primary objectives of this study is the building of dataset from natural environment, which contain large number of training examples in each defined category. For the purpose of data collection field research work is conducted. In field research work we meet the persons who expert in cow breed identification and research. After discussing the whole scenario with officials of livestock and dairy development department in Pakistan, we have selected eight cow breeds for our study. Table 1 shows the short introduction of cow breeds that proposed dataset contains. 
Table 1. Showing the detail of targeted cow breeds

\begin{tabular}{|c|c|c|}
\hline No & Bread Image with Name & Description \\
\hline 1 & - & $\begin{array}{l}\text { Cholistani breed is of medium size, having a brown body with white dots or white body with } \\
\text { small red or brown dots. Their skin is thick and loose and head of animal is medium short, ears } \\
\text { are of medium-sized, horns are usually small and thick; tail of this breed is long. }\end{array}$ \\
\hline 2 & & $\begin{array}{l}\text { Sahiwal is a medium-sized breed with fleshy and beautiful body. The body is wedged shaped } \\
\text { with loose skin, animal head is short and horns are small and thick. Hump in male cow is } \\
\text { massive while in female it is normal-sized. Usually male Sahiwal cow have small eyes, broad } \\
\text { forehead, black muzzle, and very loose sheath and dewlap. Tail of this breed is very long } \\
\text { almost touching the ground [9]. }\end{array}$ \\
\hline 3 & : & $\begin{array}{l}\text { Red Sindhi is a medium-sized breed with usually dark red skin color. The color of male cow is } \\
\text { much darker at the shoulders. In color this breed has much resemblance with "Sahiwal", in few } \\
\text { cases it is very difficult to judge the animal that either particular animal belongs to Red Sindhi } \\
\text { or Sahiwal. Horns are thick in male but female of this breed usually has thin horns, ears are fine } \\
\text { small, hump of this breed is well developed. }\end{array}$ \\
\hline 4 & 荵 & $\begin{array}{l}\text { Animals of this breed are medium-sized with different body colors, usually have white skin } \\
\text { with black patches or dots and in some cases animals have white skin with red dots or patches. } \\
\text { The head and ear of the animal of this breed are small, forehead is narrow, and horns are thick } \\
\text { and stumpy. The dewlap is small, back is straight and hump is compact. }\end{array}$ \\
\hline 5 & 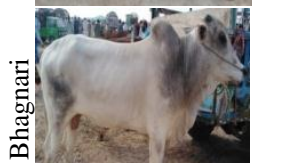 & $\begin{array}{l}\text { Body-color of this breed is usually white or light grey; usually has light black neck color. Head } \\
\text { is of medium size with short strong neck, the breed has black beautiful eyelashes. Usually have } \\
\text { small dewlap and stumpy horns, ears are small and pointed. Straight back and black tuft of the } \\
\text { tail are the prominent features of this breed to recognize it [10]. }\end{array}$ \\
\hline 6 & & $\begin{array}{l}\text { Animals of this breed are usually large and very beautiful; their skin color is white with black } \\
\text { or red patches, in some cases their skin color is completely white or black. The straight back of } \\
\text { this breed makes it easy to recognize, the head is long and narrow, the backside of Friesian cow } \\
\text { is very heavy and back legs are straight. }\end{array}$ \\
\hline 7 & & $\begin{array}{l}\text { Jersey breed have usually small body structure, skin color is usually fawn, in some cases it is } \\
\text { Redish brown or dark brown up to black coloring. Head is of long sized and face is narrowed } \\
\text { and pointed, the tail of this breed small and usually in black color [11]. }\end{array}$ \\
\hline 8 & & $\begin{array}{l}\text { Crossbreeding is a process, in which two breeds are combined to increase efficiency. In this } \\
\text { study where we are collecting images of different breeds of cow from natural environment, we } \\
\text { come to know that this process is very common now a day. Crossbreeds of cattle are more } \\
\text { common than straight breeds due to many reasons, crossbreed animals possess physical } \\
\text { characteristics of two or more breeds this factor makes them very difficult for us to place them } \\
\text { under the label of any straight breed [12]. }\end{array}$ \\
\hline
\end{tabular}

\section{b. Related work}

Saihui Hou et al., in 2018 provide a complete dataset VegFru for FGVC dataset contains 25 categories and more than 100 subcategories of vegetables and fruits. To check the validity of dataset the experimental work is done by using DCNN model [4]. Grant Van Horn et al in their paper introduce tools and methodologies to collect high quality, large scale fine-grained computer vision datasets using citizen scientists. In their research they also described that that learning algorithms based on CNN features and part localization were surprisingly robust to mislabeled training examples [13].

Similarly Zalan Raduly et al., also contributes to the FGVC problem, they use StanForddogs dataset to train deep learning models for detection of breed in a given image. They also presents two different convolutional neural network architectures, the presented architectures have been tested on an image 
classification problem i.e. recognizing dog breeds a fine-grained image recognition problem [8]. Lingyun Li et al., use (bird breeds) dataset to check the classification accuracy of fine-tuning algorithms. Lingyun Li et al., tackles the issue of over-segmentation that harm the quality of image representation, problem is frequently observed in the case of unsupervised part detection on fine-grained visual categorization [14]. Shaoyong Yu et al., in their research present a novel model for fine-grained vehicle classification that based on deep learning, their work consist of three steps, in first step they generate a dataset of vehicles in second step they use R-CNN model to detect vehicles in images and in last step they use a CNN model and joint Bayesian network to classify vehicles in fine-grained [15].

Timnit Gebru et al., proposed a graph-based crowdsourcing algorithm to automatically group visually not differentiable objects together. Using their workflow, they label 712,430 images that result in creation of a large fine-grained visual dataset of car models. In 2016 Sun et al., introduced a dataset for human skin diseases; presented dataset is the largest dataset for visual recognition of skin disease. Dataset contains 6584 images from 198 classes, for classification purposes CNN and SVM are used in this research work [16]. Ming Sun et al., introduce Dogs-in-the-Wild, a comprehensive dog species dataset for fined-grained classification. Ming Sun et al., also propose a new attention-based convolutional neural network that manages different object parts among different input images [17]. In 2017 Yi Yu et al., work on very interesting idea, they proposed a venue dataset. Venue dataset is a new type of dataset in case of FGVC, idea behind building this dataset is to discover venue from any social image. Dataset include photos of venue, its description and categories. Using this dataset they also proposed a venue discovery framework [18].

c. Cowbree vs. other datasets

Table 2 shows the detail of four datasets for FGVC, but all these four datasets are different from Cowbree. Dataset by T. Sutojo et al., is somehow similar to Cowbree but it has a very less number of training examples i.e. only 120 images belonging to 5 breeds of cow. While four targeted breeds in that particular dataset are very rare and have low domestic use. The other three datasets in table are different in nature from Cowbree, all three datasets target dog breeds.

Table 2. Detail of related datasets

\begin{tabular}{|c|c|c|c|c|}
\hline No & Author/Year & Type & Size & Used Approach \\
\hline 1 & T. Sutojo et al 2017 [19] & Cow Breeds & $\begin{array}{l}120 \text { images } \\
5 \text { categories }\end{array}$ & Content-based image retrieval model \\
\hline 2 & Omkar M Parkhi et al 2012 [20] & Cats and Dogs & $\begin{array}{l}7379 \text { images } \\
37 \text { categories }\end{array}$ & Deformable part model \\
\hline 3 & Ming Sun et al 2018 [17] & Dog Species & $\begin{array}{l}299,458 \text { images } \\
362 \text { categories }\end{array}$ & $\begin{array}{l}\text { Attention-based convolutional neural network } \\
(\mathrm{CNN})\end{array}$ \\
\hline 4 & Aditya Khosla et al 2011 [3] & Dog Species & $\begin{array}{l}22,000 \text { images } \\
120 \text { species }\end{array}$ & $\begin{array}{l}\text { Bayesian incremental algorithm for learning } \\
\text { generative models }\end{array}$ \\
\hline
\end{tabular}

\section{RESEARCH METHOD}

Figure 2 explains the hierarchy of overall research process.

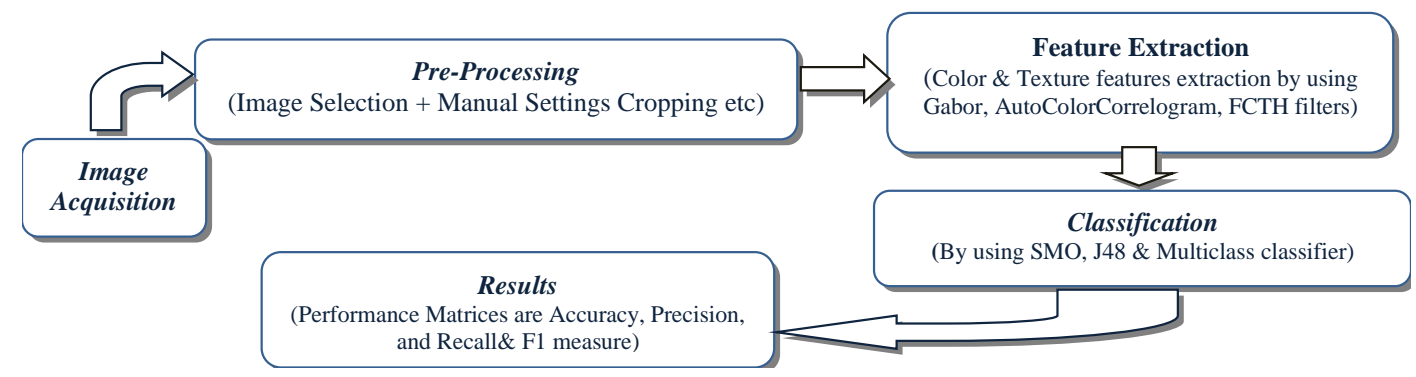

Figure 2. Overall workflow

\subsection{Image acquisition}

Image acquisition is the crucial and toughest part. More than twelve thousand images were collected from different sources. Most of the images are collected from cattle markets of Punjab. But for few rare breeds like jersey and Frisian that are popular breeds of Europe images are gathered from different web sources. This collection of images for specific breeds of the cow was a very tough task, more than six persons contributed to image acquisition part, and it took many months to gather more than 12,000 images. 


\subsection{Image selection and pre-processings}

As, this study collected enough number of images to build a dataset of different breeds of cow, however, not all of the collected images are useful. For final selection of images from overall collected images we have set following criteria:

a. Images should be collected from the natural environment, with background variations.

b. Those images should only be selected that contains a complete view of single animal, so that breed of animals can be predicted accurately.

c. Images that are of high pixel quality should be selected for the final study.

On applying defined criteria more than thirty-three hundred images of eight different cow breeds are selected for final study. In Figure 3 some examples of images that didn't meet our criteria are shown, i.e. in some pictures complete view of a single animal is not available or in some cases any other object disturbs the frame. After image selection, next task is to separate the images according to the breed of animal that image contains. This is the major and very difficult task of the whole study, this task needs very deep expert knowledge, by taking the services of officials from the livestock and dairy development department of Pakistan we placed all the selected images under their respective label i.e. breed names. Table 3 shows the exact numbers of images each label contain.
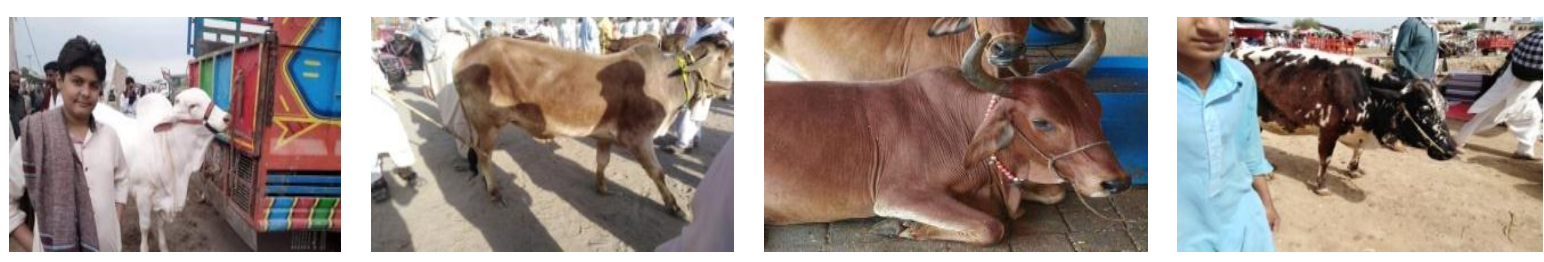

Figure 3. Images those are not fit for final study

Table 3. Showing the number of images each label contains

\begin{tabular}{lc}
\hline Name of breed & Number of images \\
\hline Cholistani & 488 \\
Sahiwal & 630 \\
Red Sindhi & 563 \\
Dhanni & 577 \\
Bhagnari & 300 \\
Friesian & 432 \\
Jersey & 413 \\
Cross-Breed & 597 \\
\hline
\end{tabular}

\subsection{Feature extraction}

After labeling the images Simple ARFF file (attribute-relation file format) of data is built by using two attributes file name and class. The process of feature extraction is carried out through WEKA image filter package. We have used threediffrent filters i.e. Auto Color Correlogram, Gabor and FCTH filter to extract the features from data [21, 22]. For feature selection main target of the study is texture and color features, as intra-class differences among the breeds are mainly body shape and color of the animal, each selected filter have some specific chracteristics. Table 4 shows the basic description of each filter and number of features extracted by each filter when it is applied on Cowbree dataset.

Table 4. Showing the basic description of filters

\begin{tabular}{|c|c|c|c|}
\hline No & Filter & Detail & \#of features extracted from data \\
\hline 1 & $\begin{array}{l}\text { Auto Color } \\
\text { Correlogram } \\
\text { Filter }\end{array}$ & $\begin{array}{l}\text { In our dataset beside texture difference among the different breeds of cow } \\
\text { the other main difference is their skin color for this particular reason we } \\
\text { have selected this filter to apply on our dataset. Auto Color Correlogram is } \\
\text { an Effective filter that robust to changes in viewing position and camera } \\
\text { zoom, it also calculates the degree of association of a picture with each } \\
\text { color [23]. }\end{array}$ & 1024 \\
\hline 2 & Gabor Filter & $\begin{array}{l}\text { A linear filter used to extract texture features from image by using Gabor } \\
\text { wavelet transform, as in our data one of the main difference among } \\
\text { different breeds is the body shape texture of the animal [24]. }\end{array}$ & 59 \\
\hline 3 & FCTH filter & $\begin{array}{l}\text { FCTH encode both color and texture information in one histogram, the size } \\
\text { of this filter is limited i.e. } 72 \text { bytes per image, so this filter is suitable for } \\
\text { use in large image datasets. Filter is very useful in our case as we have } \\
\text { large dataset and we also need both color and texture features of our } \\
\text { data [25]. }\end{array}$ & 191 \\
\hline
\end{tabular}

Bulletin of Electr Eng \& Inf, Vol. 9, No. 5, October 2020 : 1882-1889 


\section{RESULTS AND DISCUSSION}

After feature extraction next task is to apply the classifiers to get the results of three matrices i.e. accuracy, recall, and f-measure. In this work we have used three very well known state of the art classifiers for classification experiments i.e. Sequential minimal optimization (SMO), Multiclass classifier and J48. Table 5 shows the results generated by classifiers when applied to all features extracted by three image filters. From Table 4 it is clear that, SMO performs better than other classifiers and show the good accuracy value of $68.81 \%$. Now in next step in Figures 4(a-c) we draw a graph to represent the comparison of different classes (cow breeds) with respect to precision-recall and f-measure calculated by all three classifiers. This comparison helps us to find out the class that affects overall results in positive and negative way.

Table 5. Result generated by classifiers

\begin{tabular}{ccccc}
\hline Name of classifier & Precision & Recall & F Measure & Accuracy \\
\hline SMO & 0.684 & 0.688 & 0.685 & $68.81 \%$ \\
J48 & 0.570 & 0.571 & 0.575 & $57.45 \%$ \\
Multiclass & 0.556 & 0.558 & 0.556 & $55.81 \%$ \\
\hline
\end{tabular}

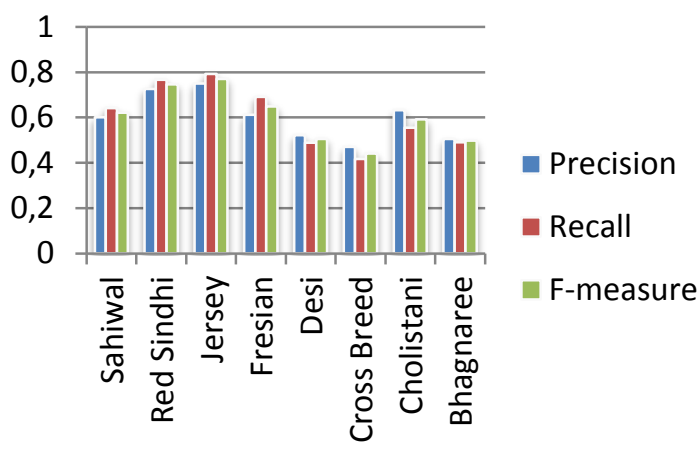

(a)

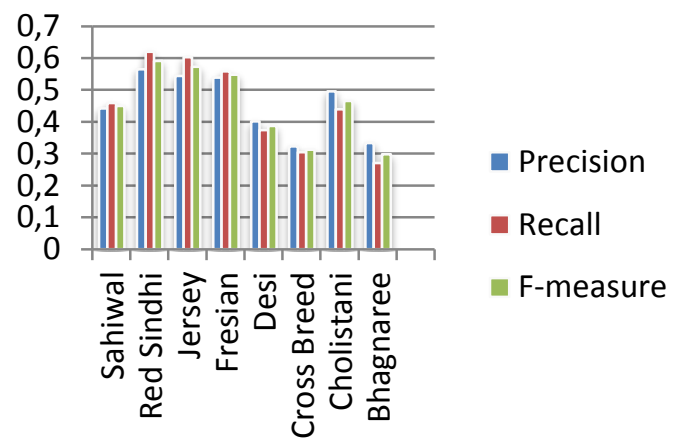

(b)

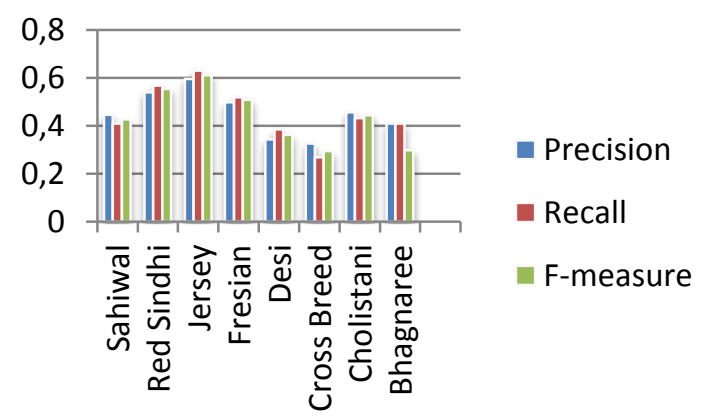

(c)

Figure 4. (a) SMO, (b) J48, (c) multiclass classifier

Statistics in graphs show that class "crossbreed" affect negatively the overall results in all three cases. Crossbreed class contains 597 examples but animal of this class differ from each other by means of texture and color. Usually in all other classes the pattern of animal in images are much similar for example in Sahiwal all the animals are of almost same color and their body structure is also quite similar but in crossbreed class both color and structure of animal is different from one another. Similarly jersey class shows the highest statistics in case of SMO and Multiclass classifier and performs positive in overall results. Class Red Sindhi perform better in case of J48. As Jersey class contains images of very similar types of animals. There is very little variation in color in all images of animals and body structure of animals in this class is quite similar. Same is case with Red Sindhi, images of animals of Red Sindhi class also shows much similarity with respect to color and body structure with each other. 


\section{CONCLUSION}

We have collected a novel domain-specific image dataset of different cow breeds that consists of 4000 images belonging to eight different categories. We also conducted an experiment by using WEKA image filter package for extracting the image features to calculate the baseline values of different matrices by using three different classifiers. As we know Fine-grained visual categorization (FGVC) refers to categorize of the objects belonging to one class but having the intra-class differences into subclasses. Collecting a fined grained dataset is a very challenging task. It requires a deep knowledge of domain.

Deep neural network has been proven to be very successful in many applications in computer vision. Therefore, we believe that one of the priorities in FGVC should be to implement techniques of deep learning such as CNN etc. If experimental work on this dataset is implemented via deep learning the results may be somehow different and the accuracy value can be increased. In the future by using this dataset, mobile app or any other model can be trained that judges the breed of cattle by taking an image as input. In countries like Pakistan, India, Bangladesh where cattle farming is a top business and the part of the life of a common villager. This type of model or application helps them to select breeds more effectively. Besides this breed detection, further this dataset can be used to train a model that can estimate the age of animal or maybe the weight of animals by using different dimensions like height of animal, shape of animal and length of horns to estimate the age of animal.

\section{REFERENCES}

[1] Y. Zhang, H. Tang, and K. Jia, "Fine-grained visual categorization using meta-learning optimization with sample selection of auxiliary data," Proceedings of the european conference on computer vision (ECCV), pp. 233-248, 2018.

[2] J. Wu, Z. Ding, L. Yao, and B. Liu, "Leveraging fine-grained labels to regularize fine-grained visual classification," in ICCMS 2019: Proceedings of the 11th International Conference on Computer Modeling and Simulation, pp. 133-136, 2019.

[3] A. Khosla, N. Jayadevaprakash, B. Yao, and L. Fei-Fei, "Novel Dataset for Fine-Grained Image Categorization," First Workshop on Fine-Grained Visual Categorization, CVPR 2011, pp. 1-2, 2011.

[4] S. Hou, Y. Feng and Z. Wang, "VegFru: A Domain-Specific Dataset for Fine-Grained Visual Categorization," 2017 IEEE International Conference on Computer Vision (ICCV), Venice, pp. 541-549, 2017.

[5] M. G. Diskin and D. G. Morris, "Embryonic and Early Foetal Losses in Cattle and Other Ruminants," Reproduction in Domestic Animals, vol. 43, no. 2, pp. 260-267, 2008.

[6] P. K. Thornton and M. Herrero, "Climate change adaptation in mixed crop-livestock systems in developing countries," Global Food Security, vol. 3, no. 2, pp. 99-107, 2014.

[7] U. Farooq, H. A. Samad, F. Sher, M. Asim, and M. Arif Khan, "Cholistan and Cholistani breed of cattle," Pakistan Veterinary Journal, vol. 30, no. 2, pp. 126-130, April 2010.

[8] Z. Ráduly, C. Sulyok, Z. Vadászi, and A. Zölde, “Dog Breed Identification Using Deep Learning,” 2018 IEEE 16th International Symposium on Intelligent Systems and Informatics (SISY), Subotica, pp. 000271-000276, 2018.

[9] Z. Rehman, M. S. Khan, and M. Aslam Mirza, "Factors affecting performance of Sahiwal cattle-A review," in Journal of Animal and Plant Sciences, vol. 24, no. 1, pp. 1-12, 2014.

[10] N. Iqbal et al., "Genomic variants identified from wholegenome resequencing of indicine cattle breeds from Pakistan," PLoS One, vol. 14, no. 4, pp. 1-21, 2019.

[11] R. O. Okeke et al., "Genetic Parameter Estimates for Milk Yield, Udder and Body Conformation Traits of Jersey Cattle in the New Derived Guinea Savannah Zone of Nigeria," Journal of Experimental Agriculture International, vol. 29, no. 1, pp. 1-5, 2018.

[12] Marvin Koger, T. J. Cunha, A. C. Warnick, "Cross-Breeding Beef Cattle," Gainesville: University of Florida Press., pp.viii-i-228, 1963.

[13] G. Van Horn et al., "Building a bird recognition app and large scale dataset with citizen scientists: The fine print in fine-grained dataset collection," 2015 IEEE Conference on Computer Vision and Pattern Recognition (CVPR), Boston, MA, pp. 595-604, 2015.

[14] L. Li, Y. Guo, L. Xie, X. Kong and Q. Tian, "Fine-grained visual categorization with fine-tuned segmentation," 2015 IEEE International Conference on Image Processing (ICIP), Quebec City, QC, pp. 20252029, 2015.

[15] S. Yu, Y. Wu, W. Li, Z. Song, and W. Zeng, "A model for fine-grained vehicle classification based on deep learning," Neurocomputing, vol. 257, pp. 97-103, 2017.

[16] X. Sun, J. Yang, M. Sun, and K. Wang, "A benchmark for automatic visual classification of clinical skin disease images," European Conference on Computer Vision, Springer, Cham, vol. 9910, pp. 206-222, 2016.

[17] M. Sun, Y. Yuan, F. Zhou, and E. Ding, "Multi-Attention Multi-Class Constraint for Fine-grained Image Recognition," European Conference on Computer Vision (ECCV), vol. 11220, pp. 834-850, 2018.

[18] Y. Yu, S. Tang, K. Aizawa, and A. Aizawa, "VenueNet: Fine-Grained Venue Discovery by Deep Correlation Learning," 2017 IEEE International Symposium on Multimedia (ISM), Taichung, pp. 288-291, 2017. 
[19] T. Sutojo, D. R. I. M. Setiadi, P. S. Tirajani, C. A. Sari, and E. H. Rachmawanto, "CBIR for classification of cow types using GLCM and color features extraction," 2017 2nd International conferences on Information Technology, Information Systems and Electrical Engineering (ICITISEE), Yogyakarta, pp. 182-187, 2017.

[20] O. M. Parkhi, A. Vedaldi, A. Zisserman, and C. V. Jawahar, "Cats and dogs," 2012 IEEE Conference on Computer Vision and Pattern Recognition, Providence, RI, pp. 3498-3505, 2012.

[21] R. Janković, "Classifying cultural heritage images by using decision tree classifiers in WEKA," Proceedings of the 1st International Workshop on Visual Pattern Extraction and Recognition for Cultural Heritage Understanding Co-Located with 15th Italian Research Conference on Digital Libraries (IRCDL 2019), Pisa, Italy, pp. 119-127, 2019.

[22] S. Karlos, N. Fazakis, K. Karanikola, S. Kotsiantis, and K. Sgarbas, "Speech recognition combining MFCCs and image features," International Conference on Speech and Computer, Springer, Cham, vol. 9811, pp. 651-658, 2016.

[23] J. Huang, S. R. Kumar, M. Mitra, W. J. Zhu, and R. Zabih, "Image indexing using color correlograms," Proceedings of IEEE Computer Society Conference on Computer Vision and Pattern Recognition, San Juan, Puerto Rico, USA, pp. 762-768, 1997.

[24] W. Jiang, T. Z. Shen, J. Zhang, Y. Hu, and X. Y. Wang, "Gabor wavelets for image processing," 2008 ISECS International Colloquium on Computing, Communication, Control, and Management, Guangzhoupp, pp. 110-114, 2008.

[25] S. A. Chatzichristofis and Y. S. Boutalis, "FCTH: Fuzzy Color and texture histogram a low level feature for accurate image retrieval," 2008 Ninth International Workshop on Image Analysis for Multimedia Interactive Services, Klagenfurt, pp. 191-196, 2008.

\section{BIOGRAPHIES OF AUTHORS}

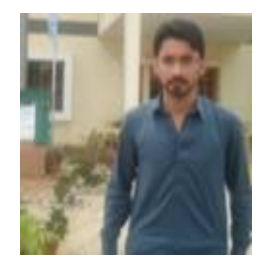

Umar Akbar Khan obtained his bachelor of science (Hons) in computer science in 2017 from The University of Sargodha, Mianwali, Pakistan. He obtained MS degree in computer sciences in 2019 from University of Lahore, Sargodha, Pakistan. His research intrest includes classification, Fined-grained visual categorization.

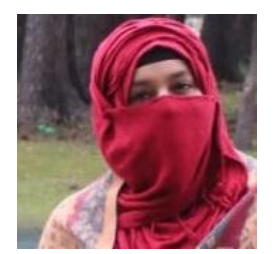

Saira Moin U. Din obtained her MSC (IT) in 2011 from University of Sargodha (UOS), Sargodha, Pakistan. She obtained MS degree in computer science degree in 2015 at University of South Asia (USA), Lahore, Pakistan. She is prursing PhD computer science at University of Sargodha, Pakistan. Her research interest includes classification, text proceasing, and social network analysis.

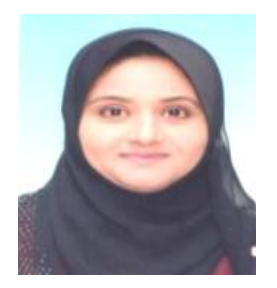

Saima Anwar Lashari obtained her bachelor of science (Hons) in computer science in 2004 at University Of Engineering \& Technology (UET), Lahore, Pakistan. She holds MSc degree in information technology in 2012 at UniversitiTun Hussein Onn Malaysia (UTHM), Malaysia. She completed her PhD in information technology at UniversitiTun Hussein Onn Malaysia (UTHM), Malaysia. Her research interest includes data mining, classification and soft set.

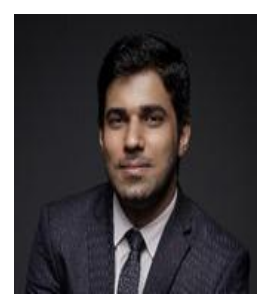

Murtaja Ali Saare is a Ph.D candidate at School of Computing, Sintok, Universiti Utara Malaysia, Kedah, Malaysia. He holds a master's degree in computer science. His research interest includes aging and cognition, e-health and Human-Centered Computing. He has published his research work inreputablescopus indexed journals.

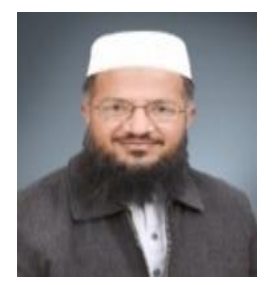

Dr. Muhammad Ilyas has done $\mathrm{PhD}$ in Computer Science from Institute for Application Oriented Knowledge Processing, Johannes Kepler University of Linz, Austria. Before That, he completed MS in Software Project Management from National University of Computer and Emerging Sciences, Lahore, Pakistan. His Research interest includes software engineering, information retrieval. 